\title{
The changes of PSII supercomplex stoichiometry in egy1 mutants are related to chlorophyll $b$ deficiency
}

\author{
M. ADAMIEC ${ }^{+}$(iD), L. MISZTAL, M. CIESIELSKA, and R. LUCIŃSKI \\ Department of Plant Physiology, Institute of Experimental Biology, Faculty of Biology, Adam Mickiewicz University, \\ Uniwersytetu Poznańskiego 6, 61-616 Poznań, Poland
}

\begin{abstract}
EGY1 is a chloroplast metalloprotease, the physiological role of which remains elusive. The changes observed in physiology and gene expression in egyl mutants indicate that lack of the protease leads to yellow-green phenotype, changes in stoichiometry in PSII complexes, and early senescence. However, the knowledge concerning the role of the EGY1 to maintain the PSII function remains elusive. The aim of our study was to gain a deeper insight into the role of EGY1 protease in maintaining proper stoichiometry of PSII complexes. We applied the blue native electrophoresis technique as well as the immunoblotting method to investigate the abundance of PSII supercomplexes and selected individual PSII apoproteins in two Arabidopsis thaliana egyl mutant lines. We also performed analyses of photosynthetic pigment content using DMSO assay. All analyses were performed in three biological replicates. Our results revealed reductions in contents of LHCII trimers and monomers in both egyl mutant lines, as well as lower accumulation levels of Lhcb1 and Lhcb2 (but not Lhcb3) apoproteins. These changes were accompanied by an increased chlorophyll $a / b$ ratio. We conclude that the observed pattern of changes in PSII stoichiometry is related to chlorophyll $b$ deficiency. This reduction of chlorophyll $b$ content is not, however, related to chlorophyllide $a$ oxygenase abundance.
\end{abstract}

Keywords: Arabidopsis thaliana; chloroplasts; EGY1; intramembrane proteases; photosystem II.

\section{Introduction}

Ethylene-dependent gravitopism-deficient and yellow green 1 (EGY1) is the first site-2 protease (S2P) discovered in plants. It is a $59.5 \mathrm{kDa}$ chloroplast, intra-membrane, ATP-independent, zinc-containing metalloprotease with experimentally confirmed proteolytic activity (Chen et al. 2005). The protein is 548 amino acids in length and comprises eight hydrophobic regions. Crucial for its proteolytic activity, the zinc-binding motif (HExxH) is located between the first and the second transmembrane regions. The second motif necessary for the proteolytic activity, namely NxxPxxxxDG, overlaps the C-terminal region of the sixth transmembrane region and a fragment of the subsequent loop (Rudner et al. 1999, Chen et al. 2005). Within the N-terminus region, the highly conserved GNLR motif was also found. The exact function of this motif unfortunately remains unknown. However, an interesting fact is that although S2P proteases are common in all living organisms, the GNLR motif has been

\section{Highlights}

- egyl mutants are chlorophyll $b$ deficient

- The stoichiometry of PSII supercomplexes in egy1 mutants is similar to the one observed in CAO-deficient plants

- EGY1 protease is indirectly involved in D1 turnover
Received 8 February 2021

Accepted 19 April 2021

Published online 13 May 2021

${ }^{+}$Corresponding author

e-mail:msolin@amu.edu.pl

\footnotetext{
Abbreviations: $\mathrm{C}_{\mathrm{x}+\mathrm{c}}$ - total carotenoids; $\mathrm{CAO}$ - chlorophyllide $a$ oxygenase; $\mathrm{Chl}$ - chlorophyll; EGY1 - ethylene-dependent gravitopismdeficient and yellow green $1 ; \mathrm{F}_{\mathrm{v}} / \mathrm{F}_{\mathrm{m}}$ - maximal quantum yield of PSII photochemistry; S2P - site-2 protease; WT - wild type.

Acknowledgements: This work was supported by the National Science Centre, Poland based on the decision number DEC-2014/15/B/ NZ3/00412.

Conflict of interest: The authors declare that they have no conflict of interest.
} 
identified only in S2P-like proteins from higher plants and cyanobacteria (Chen et al. 2005).

One of the most spectacular effects of the lack of EGY1 in A. thaliana is the characteristic yellow-green pigmentation of rosette leaves, which increases with the leaves' age. This phenotype is a consequence of a lower chlorophyll (Chl) content in the mutants, which is accompanied by impaired chloroplast biogenesis. The 4-week-old egyl A. thaliana mutants show underdevelopment of the inner chloroplast membrane system, with fewer stromal thylakoids and no grana. Additionally, fewer starch grains were observed, as well as an increased number of globular structures, which were suggested to represent plastoglobuli (Chen et al. 2005). Moreover, the longevity of the egyl leaves is significantly shorter than that in wild-type plants. This effect is considered a consequence of earlier Chl degradation as well as a reduction in the amount of soluble protein and the $F_{v} / F_{m}$ parameter with a simultaneous increase in ion leakage. Based on these observations, it was suggested that egy 1 mutants display an early-senescence phenotype (Chen et al. 2016). This suggestion was further confirmed by the fact that the darkness accelerated leaf yellowing more rapidly in egyl mutants than in wild-type plants (WT) and increased transcription of senescence-related genes $S A G 12, S A G 24, S E N 4$, and $H X K 1$ in egyl mutants (Chen et al. 2016).

More detailed studies also revealed that the reduced content of Chl, manifested by yellow-green pigmentation, is accompanied by reduced accumulation of $\mathrm{CAB}$ proteins - chlorophyll $a / b$ binding proteins forming major PSII antenna complexes (LHCII) (Chen et al. 2005). This observation was confirmed in recently published research, which indicates also that EGY1 is required for LHCII association with PSI and that the absence of protease leads to abnormal accumulation of PSII dimers and monomers (Qi et al. 2020). It has also been shown that EGY1 is a genetic enhancer mutant of var2, defective in thylakoid FtsH proteases complexes, and may participate in PsbA turnover processes (Qi et al. 2020).

In this work, we further investigate disturbed stoichiometry of PSII complexes and indicate that the observed change in the Lhcb proteins pattern coincides with analogous changes observed in a chlorophyll $b$-deficient mutant carrying a mutation in the chlorophyllide $a$ oxygenase (CAO) gene (Kim et al. 2009). Since an increase in $\mathrm{Chl} a / b$ ratio in egyl mutant lines is also observed, we hypothesize that the changes in the accumulation level of Lhcb proteins in egyl mutants may be associated with impaired activity of CAO.

\section{Materials and methods}

Plant material and growth conditions: Wild-type Arabidopsis thaliana (L.) Heynh (ecotype Columbia) (WT) and both mutant lines were grown on sphagnum peat moss and wood pulp (Jiffy peat pellets, AgroWit, Przylep, Poland) at an irradiance of $110 \mu \mathrm{mol}$ (photon) $\mathrm{m}^{-2} \mathrm{~s}^{-1}$ (white fluorescent light lamp Philips Master T-E-D $58 \mathrm{~W} / 840$ REFLEX Eco) under long-day conditions (16 h of light/ $8 \mathrm{~h}$ of darkness), a relative humidity of $70 \%$, and constant temperature of $22^{\circ} \mathrm{C}$.

The seeds of $A$. thaliana mutant lines with a T-DNA insertion in the EGY1 gene (AT5G35220) were obtained from NASC (Nottingham Arabidopsis Stock Centre, Nottingham, UK). All analyses were performed on mutant lines: SALK_134931 and SALK_061494. These lines were previously used to investigate the role of EGY1 protease in chloroplast development and were described as egyl-2 and egy 1-3, respectively (Chen et al. 2005). We decided to maintain this nomenclature; thus we named SALK_134931 egy1-2 and SALK_061494 egy1-3.

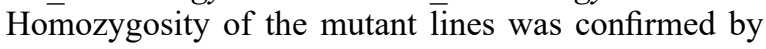
PCR technique with the following primers:

forward: 5'-CTCTACTACTAGCAGCAGCAAC-3' reverse: 5'-AGCATCTACAAATGGATACAGC-3'

T-DNA insertion (LB): 5'-CATTAAAAACGTCCGCAATGTG-3'

All analyses were performed on plants with the first flower open, which corresponds with the developmental phase 6.0 according to the BBCH scale (Boyes et al. 2001). This developmental phase was achieved on average on day 24 of culture for WT plants and on day 22 for mutants. The analyses were performed in three biological replicates. Thirty plants from each variant (WT, egyl-2, and egy1-3) were measured in each replicate.

Blue native gel electrophoresis: The thylakoid membranes corresponding to $15 \mu \mathrm{g}(\mathrm{Chl})$ were suspended to a final concentration of $1.0 \mathrm{mg}(\mathrm{Chl}) \mathrm{mL}^{-1}$ in ice-cold buffer containing $25 \mathrm{mM}$ BisTris- $\mathrm{HCl} \mathrm{pH} 7.0$ and $20 \%(\mathrm{v} / \mathrm{v})$ glycerol. Next, an equal volume of $2 \%(\mathrm{w} / \mathrm{v}) n$-dodecyl$\beta$-maltoside in the same buffer (25 mM BisTris- $\mathrm{HCl}, \mathrm{pH}$ 7.0 and $20 \%(\mathrm{v} / \mathrm{v})$ glycerol) was added and solubilization in darkness with gentle mixing was performed for $10 \mathrm{~min}$ at $4^{\circ} \mathrm{C}$. Remains of insoluble materials were removed by centrifugation $\left(18,000 \times g\right.$ for $20 \mathrm{~min}$ at $\left.4^{\circ} \mathrm{C}\right)$ and the supernatant was mixed with 0.1 volumes of sample buffer (100 mM BisTris- $\mathrm{HCl} \mathrm{pH} 7.0,0.5 \mathrm{M}$ amino-n-caproic

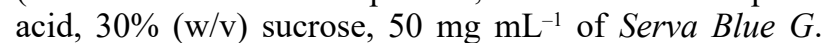
The samples were loaded onto native precast gradient gels (with a gradient 4-14\% of acrylamide) (Bio-Rad, USA). The electrophoresis was performed according to Wittig et al. (2006) with the cathode buffer containing $50 \mathrm{mM}$ tricine, $7.5 \mathrm{mM}$ imidazole, $0.02 \%(\mathrm{w} / \mathrm{v})$ Coomassie blue $G-250, \mathrm{pH} \sim 7.0$, and the anode buffer containing $7.5 \mathrm{mM}$ imidazole, $\mathrm{pH} \sim$ 7.0. During the electrophoresis, an increasing voltage was applied (from 80 to $200 \mathrm{~V}$ ) and the protein separation was performed for about $6 \mathrm{~h}$ at $4{ }^{\circ} \mathrm{C}$. To facilitate detection, the cathode buffer was replaced with a 10-times diluted cathode buffer when the front of the electrophoresis reached about three-quarters of the total distance, and to reduce the background, after the electrophoresis, the gel was incubated in water overnight at room temperature.

Protein extraction and determination of protein concentration: Protein Extraction Buffer (PEB, Agrisera, Vännäs, Sweden) was used for total protein isolation from 
$100 \mathrm{mg}$ of $A$. thaliana leaf tissue. The modified Lowry method (Lowry et al. 1951) with a Lowry DC kit (Bio-Rad, Hercules, CA, USA) was used for the determination of the extracted protein concentration.

SDS-PAGE and immunoblotting: For SDS-PAGE the modified protocol described by Laemmli (1970) was applied and $12 \%(\mathrm{w} / \mathrm{v})$ polyacrylamide gels with the addition of $6 \mathrm{M}$ urea were used. The electrophoretically separated proteins were transferred to PVDF membranes (Bio-Rad, USA), blocked for $1 \mathrm{~h}$ with $4 \%(\mathrm{w} / \mathrm{v})$ BSA (BioShop, Burlington, Canada), and incubated with appropriate primary antibodies. Next, 1-h incubation with secondary antibodies (Agrisera, Vännäs, Sweden) and a 5-min incubation with Clarity Western ECL Substrate (Bio-Rad, Hercules, CA, USA) were performed. Bands were visualized on X-ray film using an RTG Optimax $X$-ray Film Processor (Protec GmbH, Oberstenfeld, Germany). Quantification of the bands was performed using GelixOne software (Biostep GmbH, Jahnsdorf, Germany).

Antibodies: Anti-EGY1 specific polyclonal antibodies were produced in rabbits exclusively by Agrisera (Vännäs, Sweden). Highly purified (ca. 50-250 AA) N-terminal region of EGY1 from $A$. thaliana was used.

Anti-CAO antibody was purchased from Abmart Inc. (product no. $X-N P-175088.1-N$, New York, USA).

All other antibodies, namely Anti-Lhcb1, Anti-Lhcb2, Anti-Lhcb3, Anti-Lhcb4, Anti-Lhcb5, Anti-Lhcb6, antiPsbA, anti-PsbC, anti-PsbD, FtsH2/8, and Deg1 antibodies are commercially available and were purchased from Agrisera (Vännäs, Sweden). For each primary antibody linearity of immunoresponse was determined previously (Adamiec et al. 2018, 2020).

Chl and carotenoid concentration: For determination of the Chl and carotenoid concentrations DMSO assay (Hiscox and Israelstam 1979) was used. The following equations were used to determine the concentrations [ $\mu \mathrm{g} \mathrm{ml}^{-1}$ ] of chlorophyll $a(\mathrm{Chl} a)$, chlorophyll $b(\mathrm{Chl} b)$ and total carotenoids $\left(\mathrm{C}_{\mathrm{x}+\mathrm{c}}\right)$, defined as the sum of xanthophylls (x) and carotenes (c) (Sumanta et al. 2014):

Chl $a=12.47 \mathrm{~A}_{665}-3.62 \mathrm{~A}_{649}$

Chl $b=25.06 \mathrm{~A}_{649}-6.5 \mathrm{~A}_{665}$

$\mathrm{C}_{\mathrm{x}+\mathrm{c}}=\left(1,000 \mathrm{~A}_{470}-1.29 \mathrm{Chl} a-53.78 \mathrm{Chl} b\right) / 220$

The time-course photoinhibition and light stress recovery assays: The time-course analysis was performed according to Liu and Last (2017) and to our previous study (Adamiec et al. 2018, 2020). The detached leaves of the WT and mutant plants egyl-2 and egyl-3 were infiltrated with water or $1 \mathrm{mM}$ lincomycin (Sigma-Aldrich, USA) to inhibit de novo synthesis of proteins encoded on the chloroplasts' genome (i.e., PsbA polypeptide) and exposed to the irradiance of white light of $900 \mu \mathrm{mol}$ (photon) $\mathrm{m}^{-2} \mathrm{~s}^{-1}$ (white fluorescent light lamp Philips Master T-E-D $58 \mathrm{~W} / 840 \mathrm{REFLEX} \mathrm{Eco)} \mathrm{for} 5 \mathrm{~h}$. The maximum quantum yield of the PSII parameter $\left(\mathrm{F}_{\mathrm{v}} / \mathrm{F}_{\mathrm{m}}\right)$ was measured every hour after $30 \mathrm{~min}$ of leaf dark adaptation. The $\mathrm{F}_{\mathrm{v}} / \mathrm{F}_{\mathrm{m}}$ was measured using the FMS1 (Photon Systems Instruments, Brno, Czech Republic) run by Modfluor software.

For the time-course recovery treatment, the detached leaves of the WT, egy 1-1, and egy 1-3 were illuminated with an irradiance of $900 \mu \mathrm{mol}\left(\right.$ photon) $\mathrm{m}^{-2} \mathrm{~s}^{-1}$ for $5 \mathrm{~h}$ and shifted to the normal irradiance conditions $[110 \mu \mathrm{mol}$ (photon) $\mathrm{m}^{-2} \mathrm{~s}^{-1}$. Every hour part of the detached leaves was darkadapted for $30 \mathrm{~min}$ and $\mathrm{F}_{\mathrm{v}} / \mathrm{F}_{\mathrm{m}}$ was measured until no further changes in the $F_{v} / F_{m}$ were observed.

To analyze the delayed recovery of PSII, the WT and mutant plant leaves were high-light treated up to reach about $60-67 \%$ of the initial value of $F_{v} / F_{m}$. In the case of WT plants, it took about 5-h exposition of leaves to the high light, whereas for the mutant plants it only took $2.5 \mathrm{~h}$. Once the leaves reached the minimum value of the $F_{v} / F_{m}$ parameter, they were subsequently shifted to the normal light to allow recovery.

Statistical analysis: Differences in the measured parameters were analyzed for statistical significance using one-way $A N O V A$. Means were regarded as significantly different at $P<0.05$.

\section{Results}

EGY1 T-DNA insertion mutants: The physiological role of EGY1 protease in maintaining composition and proper assembly of PSII was studied in two commercially available mutants, SALK_134931 (egyl-2) and SALK_061494 (egyl-3), containing a T-DNA insertion in the gene encoding the protease AT5G35220. To verify the number and location of T-DNA insertions in the egyl-2 and egy 1-3 mutants, the PCR technique was used with different combinations of primers for WT, egyl-2, and egy 1-3. In the egy 1-2 mutant, our analysis indicated the presence of two T-DNA insertions, localized in the first intron, while in the egyl-3, only one T-DNA insertion, located in the second exon, was found (Fig. $1 A, B$ ). The absence of the EGY1 protease in both egyl-2 and egyl-3 mutant lines was confirmed with Western blot (Fig. 1C).

Composition of thylakoid membrane complexes and pigment content: We compared the oligomeric state of thylakoid membrane complexes in WT plants and both egyl mutant lines using the blue native electrophoresis technique. In comparison to WT plants both egyl mutants were characterized by lower intensities of bands representing PSII supercomplexes. The bands corresponding to the $\mathrm{C}_{2} \mathrm{~S}_{2} \mathrm{M}_{2}$ PSII as well as the $\mathrm{C}_{2} \mathrm{~S}_{2} \mathrm{M}$ and $\mathrm{C}_{2} \mathrm{~S}$ in both egy 1-2 and egy 1-3 mutant lines were about 50\% less abundant than that in the WT plants, while the abundance of the band corresponding to $\mathrm{C}_{2} \mathrm{~S}_{2}$ was approximately $30 \%$ lower than that in WT plants (Fig. 2). Significantly reduced abundance, to approximately $60 \%$, was also observed in bands corresponding to LHCII trimers and monomers. A statistically significant increase was, in turn, observed in bands corresponding to PSII monomer and ATP synthase. In the egy 1-2 mutant line, the abundance of PSII monomer increased to about $150 \%$ and the intensity of the band representing ATP synthase to $140 \%$ and in egyl-3 the 
accumulation level of ATP synthase increased to about $155 \%$ while the accumulation of the PSII monomer reached about $150 \%$ of the value observed in WT plants. The intensity of the band corresponding to the LHCII assembly complexes in both egyl mutant lines remained at a level similar to the one observed in WT plants (Fig. 2).
Accumulation levels of selected PSII apoproteins: In addition to the analysis of changes in abundance of thylakoid membrane complexes using the blue native electrophoresis method, the comparative analysis of accumulation levels of selected apoproteins forming the PSII complex was performed. Significant changes in

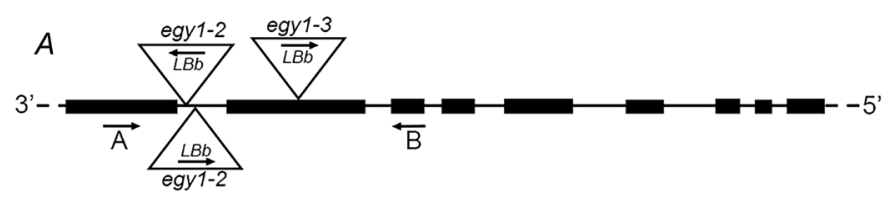

$B$
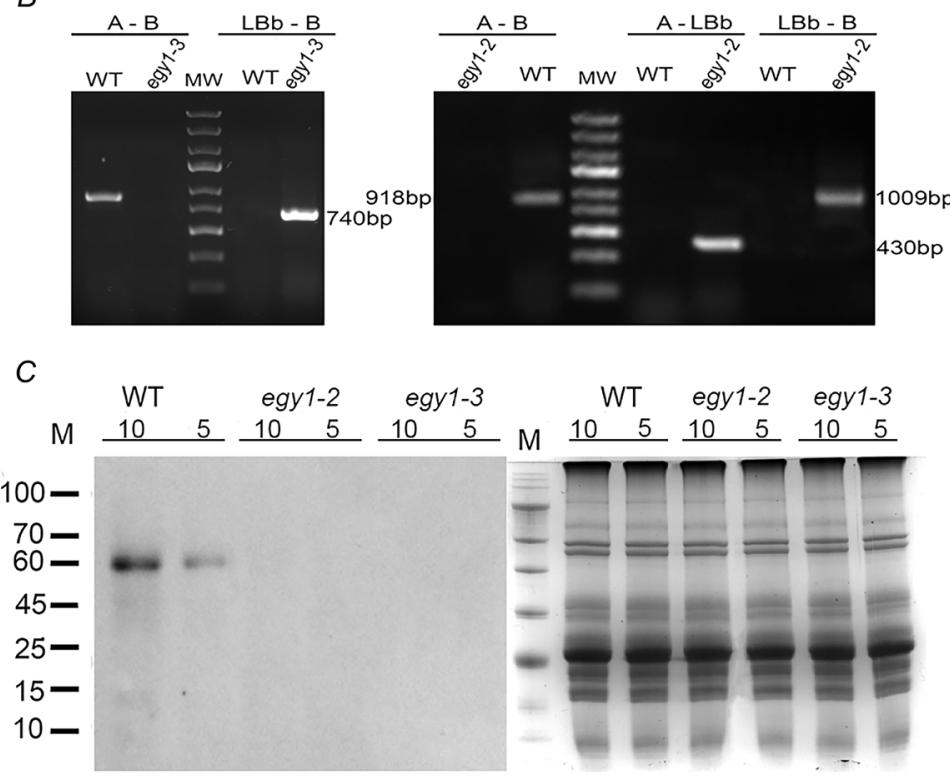

Fig. 1. Identification of egyl-2 and egy1-3 mutant lines. (A) Schematic diagram of the Arabidopsis thaliana EGY1 gene. The black boxes represent exons and introns are shown as black lines. The triangles show the locations of T-DNA insertions. The arrows mark the annealing sites of the primers used for PCR analysis. $(B)$ Confirmation of the homozygosity of the egyl-2 and egyl-3 mutants. Amplification was performed using the $\mathrm{A}, \mathrm{B}$, and $\mathrm{LBb}$ primers as indicated in Fig. $1 A$. (C) Immunoblot analysis of the abundance of EGY1 protein in the wild-type plants (WT) and both mutant lines. Samples containing 10 and $5 \mu \mathrm{g}$ of total leaves protein were separated by SDS-PAGE and transferred to PVDF membranes. Subsequently the immunoblot assay with use of anti-EGY1 primary antibodies was applied. Coomassie staining was used as an equal loading control.

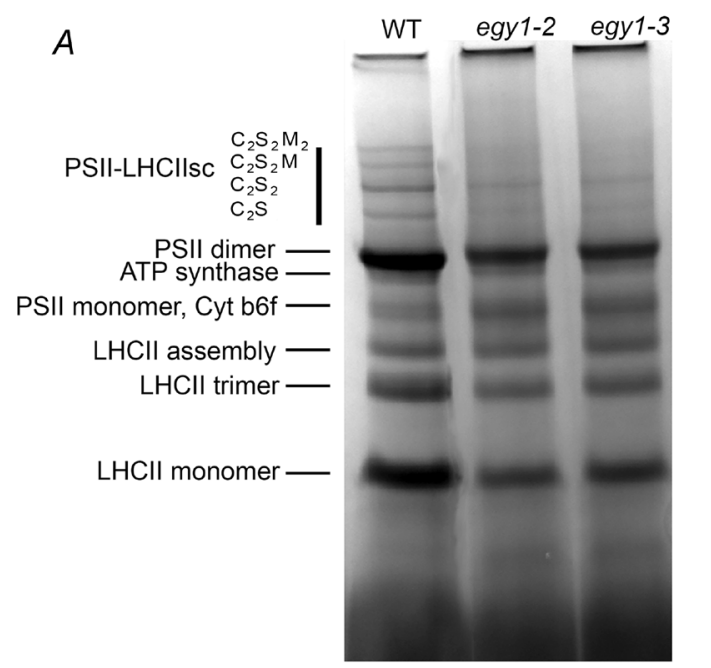

$B$

\begin{tabular}{llccc}
\hline & & WT [\%] & egy1-2 [\%] & egy1-3 [\%] \\
\hline 1 & PSII-LHCII $\mathrm{C}_{2} \mathrm{~S}_{2} \mathrm{M}_{2}$ & 100 & $63^{*} \pm 5$ & $50^{*} \pm 8$ \\
2 & PSII-LHCII $\mathrm{C}_{2} \mathrm{~S}_{2} \mathrm{M}$ & 100 & $55^{*} \pm 7$ & $55^{*} \pm 5$ \\
3 & PSII-LHCII $\mathrm{C}_{2} \mathrm{~S}_{2}$ & 100 & $70^{*} \pm 4$ & $75^{*} \pm 8$ \\
4 & PSII-LHCII $\mathrm{C}_{2} \mathrm{~S}$ & 100 & $58^{*} \pm 6$ & $55^{*} \pm 4$ \\
5 & PSII dimer & 100 & $90 \pm 7$ & $86 \pm 8$ \\
6 & ATP synthase & 100 & $140^{*} \pm 7$ & $155^{*} \pm 7$ \\
7 & PSII monomer & 100 & $155^{*} \pm 9$ & $150^{*} \pm 8$ \\
8 & LHCII assembly & 100 & $115 \pm 6$ & $100 \pm 8$ \\
9 & LHCII trimer & 100 & $63^{*} \pm 9$ & $60^{*} \pm 7$ \\
10 & LHCII monomer & 100 & $68^{*} \pm 7$ & $60^{*} \pm 6$ \\
\hline
\end{tabular}

Fig. 2. Blue-native PAGE analysis of thylakoid membranes complexes. The thylakoid membranes were isolated from WT, egy1-2, and egyl-3 plants and solubilized with $1 \%(\mathrm{w} / \mathrm{v}) n$-dodecyl- $\beta$-maltoside. Thylakoid membrane complexes $(15 \mu \mathrm{g}$ of total protein) were loaded onto gel and separated by BN-PAGE. Panel $(A)$ presents the representative gel and the results of densitometry of thylakoid membranes complexes are presented in panel $(B)$. ' \pm ' indicates the SD determined in the analysis of samples obtained from four biological replicates. The asterisks indicate statistically significant differences between the WT and individual mutants. 
proteins forming the major peripheral antenna complexes (LHCII) Lhcb1 and Lhcb2 were observed. In both egy1 mutant lines, the abundance of Lhcb1 and Lhcb2 decreased to approximately 75 and $70-60 \%$, respectively, in comparison to WT plants. The abundance of Lhcb3 in mutant lines remained, however, unchanged (Fig. 3). Decreased abundance was also observed for apoproteins Lhcb4 and Lhcb6 forming minor peripheral antenna complexes. The Lhcb4 apoprotein level decreased to $71 \%$ in the egyl-2 mutant line and to $60 \%$ in the egyl-3 mutant line, whereas the accumulation of Lhcb6 apoprotein decreased to 75 and $60 \%$ in egyl-2 and egyl-3 mutants, respectively. No statistically significant changes were observed in the abundance of Lhcb5 (Fig. 3). A decrease in abundance was observed for PsbC apoprotein, associated with an inner PSII antenna - the CP43 complex. In the egy 1-2 mutant line, the level of PsbC decreased to 64\% and to $71 \%$ in egyl-3 (Fig. 4). The accumulation levels of PsbA and PsbD apoproteins, forming the PSII reaction center, were also investigated. A decrease in abundance of PsbD to $56 \%$ in egy $1-2$ and to $57 \%$ in egy $1-3$ was observed. The level of PsbA apoprotein, however, significantly increased and reached $262 \%$ in the egyl-2 mutant line and $259 \%$ in egyl-3 (Fig. 4).

Pigment content and $\mathrm{Chl} \boldsymbol{a} / \boldsymbol{b}$ ratio: Pigment concentrations in WT plants, egy 1-2, and egy 1-3 mutant lines were measured on plants with the first flower open. This ontogenetic phase is described by Boyes et al. (2001) as developmental phase 6.0 of $A$. thaliana. In both mutant lines, the $\mathrm{Chl}$ and carotenoid concentrations significantly decreased. The Chl $a$ content was reduced to approximately $56 \%$ in both mutant lines, but a significantly larger decrease was observed in Chl $b$ concentration, which was about one third of the value observed in WT plants. The observed changes in Chl $a$ and $b$ concentration resulted in a significant increase in the $\mathrm{Chl} a / b$ ratio, from 1.8 in WT plants to an average of 3.02 in egyl-2 and 2.98 in egyl-3 mutant lines. In both egyl mutant lines, a significant decrease of carotenoid content was also observed, to approximately $60 \%$ in relation to WT plants (Table 1). The observed increase in Chl $a / b$ ratio in egyl mutant lines prompted us to investigate the abundance of chlorophyllide $a$ oxygenase (CAO), which is crucial for $\mathrm{Chl} b$ biosynthesis. However, no statistically significant differences in accumulation level of CAO, between egyl mutants and WT plants, were observed (Fig. 5).

PSII sensitivity to photoinhibitory conditions and recovery rate: The observed changes in PSII supercomplex composition prompted us to investigate the role of EGY1 protein in the sensitivity of PSII to photoinhibitory conditions. The leaves of the WT and mutant plants were subjected to a time-course experiment with lincomycin treatment to avoid the synthesis of proteins encoded on the chloroplasts' genome. In both egyl mutant lines, a more rapid decrease in the $F_{v} / F_{m}$ parameter was observed both in the presence and in the absence of lincomycin, indicating increased sensitivity of PSII to photodamage (Fig. 6A,B). What is more, the recovery of the egyl mutants was also significantly slower than that in WT plants and the $F_{v} / F_{m}$ parameter did not reach the initial value (Fig. 6C). To investigate whether the observed delayed recovery in the mutants was a consequence of excessive PSII photoinactivation, the mutants and WT plants were exposed to high irradiance sufficient to induce similar photoinhibition and then allowed to recover under

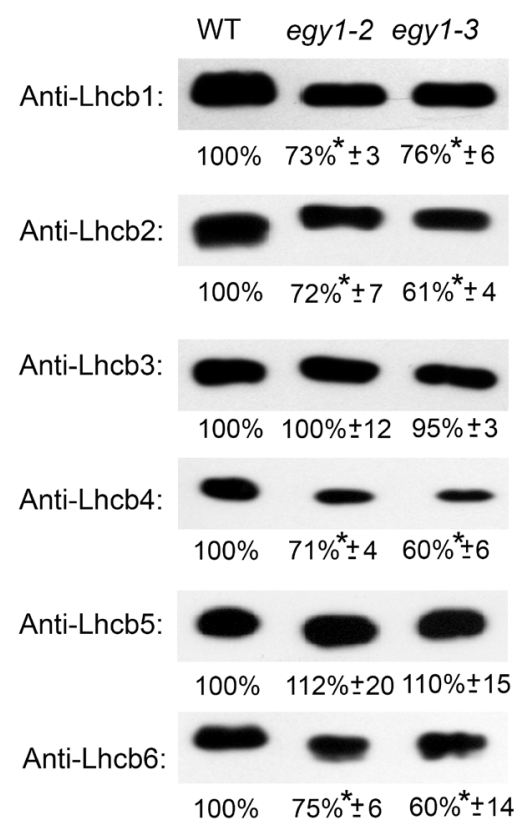

Fig. 3. Immunoblot analysis of the contents of Lhcb1-6 apoproteins in wild-type (WT), egy 1-2, and egy 1-3 mutants. Total protein $(3 \mu \mathrm{g})$ from each sample was subjected to immunoblotting analysis with specific primary antibodies. Quantification of the blots was performed using GelixOne software. The individual apoprotein content of the mutants was quantified as a percentage of the antibody signal strength in the WT (100\%). ' \pm ' indicates the SD calculated from the analysis of samples from the four biological replicates. The asterisks indicate statistically significant differences between the WT and individual mutants.

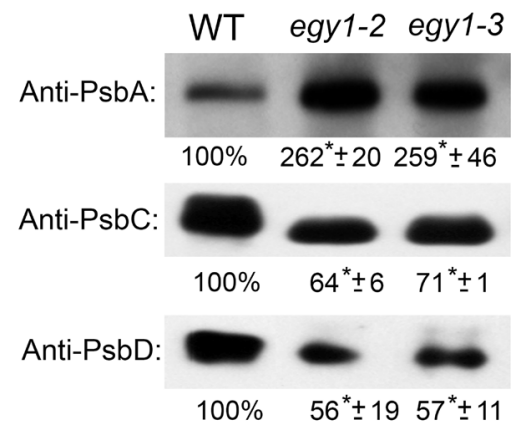

Fig. 4. Immunoblot quantification of PsbA, PsbC, and PsbD apoproteins in wild-type (WT), egy 1-2, and egy 1-3. Total protein $(2 \mu \mathrm{g})$ was immunologically analyzed using an appropriate primary antibody. GelixOne software was used to quantify the blots. ' \pm ' indicates the SD determined in the analysis of samples obtained from three biological replicates. 
Table 1. Comparison of the chlorophyll and carotenoid contents in leaves in the wild-type (WT) plants and egyl-2 and egyl-3 mutant lines in normal light conditions. ' \pm ' indicates the SD calculated from the analysis of four biological replicates (30 plants each). '*' indicates statistically significant differences between the WT and individual mutants.

\begin{tabular}{llll}
\hline & WT & egyl-2 & egyl-3 \\
\hline Chlorophyll $a\left[\mu \mathrm{g} \mathrm{g}^{-1}(\mathrm{FM})\right]$ & $485 \pm 27$ & $286 \pm 20^{*}$ & $296 \pm 11^{*}$ \\
Chlorophyll $b\left[\mu \mathrm{g} \mathrm{g}^{-1}(\mathrm{FM})\right]$ & $269 \pm 15$ & $95 \pm 12^{*}$ & $99 \pm 6^{*}$ \\
Chlorophyll $a / b$ & $1.80 \pm 0.14$ & $3.01 \pm 0.14^{*}$ & $2.98 \pm 0.14^{*}$ \\
Carotenoids $\left[\mu \mathrm{g} \mathrm{g}^{-1}(\mathrm{FM})\right]$ & $94 \pm 8$ & $58 \pm 5^{*}$ & $60 \pm 8^{*}$ \\
\hline
\end{tabular}

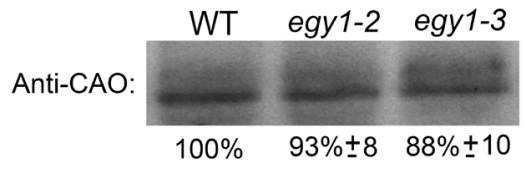

Fig. 5. Immunoblot quantification of CAO protein in wildtype (WT), egyl-2, and egyl-3. Total protein $(10 \mu \mathrm{g})$ was immunologically analyzed using an appropriate primary antibody. GelixOne software was used to quantify the blots. ' \pm ' indicates the SD determined in the analysis of samples obtained from tree biological replicates.

normal light conditions. It was observed that also under these conditions both the egyl mutant lines showed slower recovery than that of WT plants, which is further evidence for impaired PSII repair cycle in the mutants (Fig. 6D). Taking into account the results indicating a slower PSII recovery rate after exposure to photoinhibitory conditions, we decided to investigate the accumulation level of selected proteases involved in the PSII-repair cycle using an immunoblot technique. Surprisingly, we observed increased intensity of the band corresponding to $\mathrm{FtsH} 2 / 8$ subunits of the FtsH protease complex, which was about $234 \%$ in egy $1-2$ and about $246 \%$ in egy $1-3$ compared with the value observed in WT plants. However, the abundance of Deg1 protease significantly decreased, namely to $61 \%$ in egyl-2 and 65\% in the egyl-3 mutant line (Fig. 7).

\section{Discussion}

The PCR analysis confirmed the homozygosity of the analyzed mutant lines and allowed us to establish an approximate location of T-DNA insertion. In egy1-2, two insertions of T-DNA are present and both of them are located in the first intron. In egy 1-3, the insertion was found in the second exon (Fig. 1). These results are consistent with previous findings (Chen et al. 2005). Moreover, the EGY1 protease was undetectable in both mutant lines.

In both egyl mutant lines, the reduction of the major peripheral antenna of LHCII trimers and monomers was observed (Fig. 2). The result is consistent with previous reports concerning changes in PSII complexes stoichiometry related to the lack of EGY1 protease (Chen et al. 2005, Qi et al. 2020). However, the decrease was visible not only in the lower abundance of bands representing LHCII trimers and monomers but also in a lower level of PSII supercomplexes. These supercomplexes are com- posed of two main moieties: the core complex, which functions as a homodimer $\left(\mathrm{C}_{2}\right)$, and, associated with the homodimer, the peripheral antenna system composed of six different Lhcb proteins. The minor antennae occur as monomers and are formed by Lhcb4, Lhcb5, and Lhcb6 proteins. The major light-harvesting complexes (LHCII) are homo- or heterotrimers composed of Lhcb1, Lhcb2, and Lhcb3 proteins. The different forms of LHCII trimers vary in affinity to the PSII core complex. The homodimer $\left(\mathrm{C}_{2}\right)$ with two strongly associated LHCII (S-LHCII) Lhcb4 and Lhcb5 proteins (two copies of each) forms the $\mathrm{C}_{2} \mathrm{~S}_{2}$ supercomplex. This complex, in turn, binds two moderately associated LHCII (M-LHCII) together with two copies of Lhcb6 and constitutes the $\mathrm{C}_{2} \mathrm{~S}_{2} \mathrm{M}_{2}$ supercomplex (Cao et al. 2018). In both egyl mutant lines, a lower abundance of all bands corresponding to PSII supercomplexes, namely $\mathrm{C}_{2} \mathrm{~S}_{2} \mathrm{M}_{2}, \mathrm{C}_{2} \mathrm{~S}_{2} \mathrm{M}, \mathrm{C}_{2} \mathrm{~S}_{2} \mathrm{C}_{2} \mathrm{~S}$, was observed. These results are consistent with our analysis of the accumulation level of individual Lhcb proteins, which revealed a decreased accumulation level of Lhcb1 and Lhcb2 apoproteins. These apoproteins are the main components of the major peripheral antenna and constitute $89 \%$ of total LHCII protein content (Luciński and Jackowski 2006). Simultaneously, a decrease in abundance of Lhcb4 and Lhcb6 proteins was observed. These apoproteins are components of the minor peripheral antenna CP29 and CP24, respectively. The accumulation level of the third component of LHCII complexes - the Lhcb3 protein and the amount of Lhcb5 apoprotein, which constitutes the CP26 minor peripheral antenna, remain similar to those observed in WT plants. It can be assumed that the observed decrease in the abundance of PSII-LHCII supercomplexes are at least partially related to the lower accumulation of inner antennae proteins Lhcb4 and Lhcb6. Both apoproteins were previously proven to be crucial for the association of M-LHCII trimers to $\mathrm{C}_{2} \mathrm{~S}_{2}$ core (Kovács et al. 2006, de Bianchi et al. 2011). Also, observed changes in $\mathrm{Chl} a / b$ ratio remain in agreement with the observed stoichiometry of PSII supercomplexes of the egyl mutant. Chl $a$ is present in both the PSII core and PSII antenna while Chl $b$ occurs predominantly in peripheral antenna proteins; thus $\mathrm{Chl} a / b$ ratio is an indicator of PSII antenna size (Tanaka et al. 2001). The decreases in a Chl content and yellow-green phenotype were previously mentioned as symptoms of early senescence of the egyl mutants (Chen et al. 2016). However, the observed changes in the stoichiometry of PSII complexes and increase in Chl $a / b$ ratio are inconsistent with changes observed during 


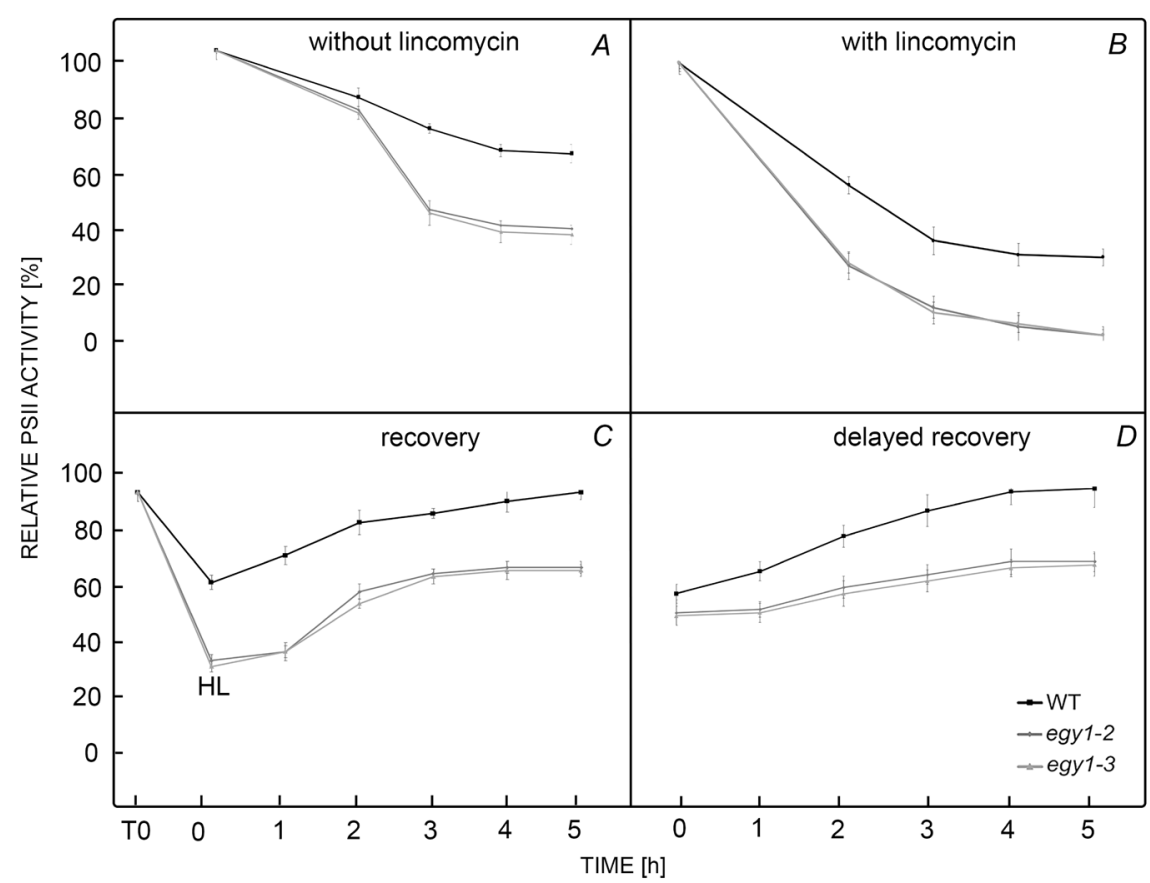

Fig. 6. The time-course changes of the maximal PSII efficiency $\left(\mathrm{F}_{\mathrm{v}} / \mathrm{F}_{\mathrm{m}}\right)$. The measurements were performed on detached leaves of plants grown under irradiance of $110 \mu \mathrm{mol}$ (photon) $\mathrm{m}^{-2} \mathrm{~s}^{-1}$. The leaves were treated with water $(A)$ or $1 \mathrm{mM}$ lincomycin $(B)$ and placed under irradiance of $900 \mu \mathrm{mol}\left(\right.$ photon) $\mathrm{m}^{-2} \mathrm{~s}^{-1}$. The measurements of $\mathrm{F}_{\mathrm{v}} / \mathrm{F}_{\mathrm{m}}$ were performed after 2, 3, 4, and $5 \mathrm{~h}$ of exposure to high irradiance. The average 'absolute' initial value of $\mathrm{F}_{\mathrm{v}} / \mathrm{F}_{\mathrm{m}}$ was 0.798 for WT plants, 0.802 for egyl-2, and 0.803 for egyl-3. The changes in $\mathrm{F}_{\mathrm{v}} / \mathrm{F}_{\mathrm{m}}$ in recovery phase $(C)$ were performed on leaves exposed to $900 \mu \mathrm{mol}$ (photon) $\mathrm{m}^{-2} \mathrm{~s}^{-1}$ for $4 \mathrm{~h}$ (HL point) and transferred again to $110 \mu \mathrm{mol}$ (photon) $\mathrm{m}^{-2} \mathrm{~s}^{-1}$. T0 means the 'absolute' initial value of $\mathrm{F}_{\mathrm{v}} / \mathrm{F}_{\mathrm{m}}$. The delayed recovery $(D)$ was measured on leaves previously high light-treated up to about $60 \%$ (mutant plants) $-67 \%$ (WT) of the initial $\mathrm{F}_{\mathrm{v}} / \mathrm{F}_{\mathrm{m}}$ value and then shifted to the normal light conditions to allow recovery. The $F_{v} / F_{m}$ measurements were taken every hour until full recovery of PSII was observed.

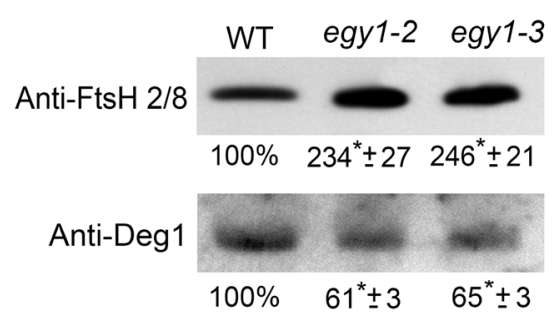

Fig. 7. Immunoblot analysis of $\mathrm{FtsH} 2 / 8$ and $\mathrm{Deg} 1$ proteins in wild-type (WT) plants and egyl-2, and egyl-3 mutant lines. The equal total proteins form Arabidopsis thaliana leaves $(5 \mu \mathrm{g})$ were separated by SDS-PAGE and subjected to immunoblot analysis with specific primary antibody. The GelixOne software was used for blots quantification. The experiment was performed in four biological replicates. ' \pm ' indicates the SD. The asterisks indicate the statistically significant differences between the WT and individual mutants.

A. thaliana senescence. The natural senescence of A. thaliana chloroplasts results in a decrease of $\mathrm{Chl} a / b$ ratio since LHCII complexes remain relatively stable in thylakoid membranes (Nath et al. 2013). The pattern of changes in abundance of Lhcb1-6 proteins observed in the analyzed mutant is similar rather to the one observed in $\mathrm{Chl} b$-deficient mutants of $A$. thaliana than to changes occurring during senescence. Chl $b$ is synthesized by oxidation of a methyl group on the B ring of the porphyrin molecule to a formyl group by chlorophyllide $a$ oxygenase (CAO). The deletion in the gene encoding CAO disables the Chl $b$ synthesis and leads to decreased abundance of Lhcb1, Lhcb2, Lhcb4, and Lhcb6. The accumulation levels of Lhcb3 and Lhcb5 remain unchanged (Kim et al. 2009). For these reasons, we decided to verify the CAO content in egyl mutants. Finally, we found that the abundance of CAO is similar in egyl mutants lines and WT plants (Fig. 5), thus the observed deficiency in $\mathrm{Chl} b$ seems not to be a result of changes in CAO accumulation level. It could be hypothesized, however, that the observed changes in the Chl $a / b$ ratio and Lhcb proteins could be - at least the effect of disturbed CAO activity. Further research is needed to investigate this issue. The reduced antenna size associated with $\mathrm{Chl} b$ deficiency may be a cause of increased sensitivity of egyl mutants to photoinhibition since this feature occurred also in Chl $b$-deficient mutants (Kim et al. 2009). An important role of LHCII in photostability of PSII was previously documented and is associated with destabilization of the PSII donor active site (Havaux and Tardy 1997).

In our experiments performed on plants at the 6.0 developmental phase, significant overaccumulation of PsbA protein was also observed. This result was consistent with the overaccumulation of the PSII monomer. 
However, in previous research performed by Qi et al. (2020) on two-week-old Arabidopsis thaliana plants, the observed increase in PSII monomer and PSII dimer contents was not linked to the increased PsbA accumulation level. A lower accumulation level of PsbC apoprotein, associated with an inner PSII antenna - the CP43 complex, observed in our analysis - has not been previously reported. The inconsistencies in the quantification of individual PSII apoproteins obtained from two-week- and four-weekold plants may result from both the developmental phase and the growth conditions. Since previously described experiments were performed on plants grown in continuous light conditions, while in our analysis long-day conditions (16 h of light $/ 8 \mathrm{~h}$ of darkness) were used.

The different development phases and light conditions also influenced the abundance of proteases crucial for D1 turnover. In two-week-old egyl mutants, the abundance of FtsH2 protein was similar to that one observed in WT plants (Qi et al. 2020), however, our results revealed a significant overaccumulation of $\mathrm{FtsH} 2 / 8$ subunits of the FtsH complex. On the other hand, we observed also a significant decrease in the abundance of Deg1 protein, which was documented to play an important role in maintaining proper PSII functioning in photoinhibitory conditions. The Deg1 protease cleaves lumen-exposed loops of PsbA and cooperates with the FtsH protease complex in the degradation of PsbA protein during the process of PSII repair (Kapri-Pardes et al. 2007). The PsbA protein was, however, proven to be efficiently degraded in egyl mutants (Qi et al. 2020), so other factors leading to increased susceptibility to photoinhibition of egyl mutants should be considered. Since our analysis indicates that recovery of egyl mutants after photoinhibitory conditions is significantly slower than that in the WT plants, it cannot be excluded that lack of EGY1 leads to slower reassembly of PSII complexes after degradation of photodamaged PsbA. This finding is also another argument from our experiments that supports Qi's thesis (Qi et al. 2020) that EGY1 is an important player in chloroplast development regulation (which acts synergistically with FtsH heterocomplex). Moreover, it seems that the lack of EGY1 leads to huge abnormalities in the protease content of chloroplasts at the late vegetative phase of $A$. thaliana development.

In conclusion, observed changes in the stoichiometry of PSII complexes as well as the yellow-green phenotype of egyl mutants and observed decrease in the chlorophyll content are partially a result of chlorophyll $b$ deficiency. The pattern of changes in abundance of individual PSII apoproteins is consistent with the one observed in chlorophyll $b$-deficient Arabidopsis thaliana mutants. The lower chlorophyll $b$ content is not, however, a result of CAO abundance and the role of EGY1 protease in maintaining proper chlorophyll $b$ content remains unclear.

\section{References}

Adamiec M., Misztal L., Kasprowicz-Maluśki A., Luciński R.: EGY3: homolog of S2P protease located in chloroplasts. Plant Biol. 22: 735-743, 2020.
Adamiec M., Misztal L., Kosicka E. et al.: Arabidopsis thaliana egy2 mutants display altered expression level of genes encoding crucial photosystem II proteins. - J. Plant Physiol. 231: 155-167, 2018.

Boyes D.C., Zayed A.M., Ascenzi R. et al.: Growth stagebased phenotypic analysis of Arabidopsis: A model for high throughput functional genomics in plants. - Plant Cell 13: 1499-1510, 2001

Cao P., Su X., Pan X. et al.: Structure, assembly and energy transfer of plant photosystem II supercomplex. - BBABioenergetics 1859: 633-644, 2018.

Chen C., Wang J., Zhao X.: Leaf senescence induced by EGY1 defection was partially restored by glucose in Arabidopsis thaliana. - Bot. Stud. 57: 5, 2016.

Chen G., Bi Y.R., Li N.: EGY1 encodes a membrane-associated and ATP-independent metalloprotease that is required for chloroplast development. - Plant J. 41: 364-375, 2005.

de Bianchi S., Betterle N., Kouril R. et al.: Arabidopsis mutants deleted in the light-harvesting protein Lhcb4 have a disrupted photosystem II macrostructure and are defective in photoprotection. - Plant Cell 23: 2659-2679, 2011.

Havaux M., Tardy F.: Thermostability and photostability of photosystem II in leaves of the chlorina- 2 barley mutant deficient in light-harvesting chlorophyll $a / b$ protein complexes. - Plant Physiol. 113: 913-923, 1997.

Hiscox J.D., Israelstam G.F.: A method for the extraction of chlorophyll from leaf tissue without maceration. - Can. J. Bot. 57: 1332-1334, 1979.

Kapri-Pardes E., Naveh L., Adam Z.: The thylakoid lumen protease Deg1 is involved in the repair of photosystem II from photoinhibition in Arabidopsis. - Plant Cell 19: 1039-1047, 2007.

Kim E.H., Li X.P., Razeghifard R. et al.: The multiple roles of light-harvesting chlorophyll $a / b$-protein complexes define structure and optimize function of Arabidopsis chloroplasts: A study using two chlorophyll $b$-less mutants. BBA-Bioenergetics 1787: 973-984, 2009.

Kovács L., Damkjær J., Kereïche S. et al.: Lack of the lightharvesting complex CP24 affects the structure and function of the grana membranes of higher plant chloroplasts. - Plant Cell 18: 3106-3120, 2006.

Laemmli U.K.: Cleavage of structural proteins during the assembly of the head of bacteriophage T4. - Nature 227: 680-685, 1970.

Liu J., Last R.L.: A chloroplast thylakoid lumen protein is required for proper photosynthetic acclimation of plants under fluctuating light environments. - P. Natl. Acad. Sci. USA 114: E8110-E8117, 2017.

Lowry O.H., Rosebrough N.J., Farr L.A., Randall R.J.: Protein measurement with the folin phenol reagent. - J. Biol. Chem. 193: 265-275, 1951.

Luciński R., Jackowski G.: The structure, functions and degradation of pigment-binding proteins of photosystem II. Acta Biochim. Pol. 53: 693-708, 2006.

Nath K., Phee B.K., Jeong S. et al.: Age-dependent changes in the functions and compositions of photosynthetic complexes in the thylakoid membranes of Arabidopsis thaliana. Photosynth. Res. 117: 547-556, 2013.

Qi Y., Wang X., Lei P. et al.: The chloroplast metalloproteases VAR2 and EGY1 act synergistically to regulate chloroplast development in Arabidopsis. - J. Biol. Chem. 295: 10361046, 2020.

Rudner D.Z., Fawcett P., Losick R.: A family of membraneembedded metalloproteases involved in regulated proteolysis of membrane-associated transcription factors. - P. Natl. Acad. Sci. USA 96: 14765-14770, 1999. 
Sumanta N., Haque C.I., Nishika J., Suprakash R.: Spectrophotometric analysis of chlorophylls and carotenoids from commonly grown fern species by using various extracting solvents. - Res. J. Chem. Sci. 4: 63-69, 2014.

Tanaka R., Koshino Y., Sawa S. et al.: Overexpression of chlorophyllide a oxygenase (CAO) enlarges the antenna size of photosystem II in Arabidopsis thaliana. - Plant J. 26: 365-373, 2001.

Wittig I., Braun H.-P., Schägger H.: Blue native PAGE. - Nat. Protoc. 1: 418-428, 2006.

(C) The authors. This is an open access article distributed under the terms of the Creative Commons BY-NC-ND Licence. 